\title{
A Simple Mathematical Method to Predict the Course of a First Episode of Liver Viral Cytolysis
}

\author{
NICOLA DIOGUARDI ${ }^{\mathrm{a}, \mathrm{b}, *}$, ANTONIO BARBIERI $^{\mathrm{b}, \mathrm{e}}$, RENATO DACCO $^{\mathrm{c}}$ and FABIO GRIZZI ${ }^{\mathrm{b}, \mathrm{d}}$ \\ ${ }^{a}$ Direzione Scientifica. Istituto Clinico Humanitas, Via Manzoni 56, 20089 Rozzano, Milan, Italy; ${ }^{\mathrm{b}}$ Centro di Medicina Teoretica, ${ }^{* *}$ \\ University of the Study, 20100 Milan, Italy; ${ }^{\mathrm{C}}$ Ospedale S. Giuseppe, 20100 Milan, Italy; ${ }^{\mathrm{d}}$ Istituto Clinico Humanitas, 20089 Rozzano,

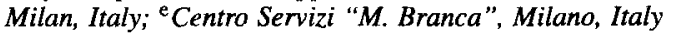

(Received 9 July 1998; Revised 28 October 1998; In final form 8 December 1998)

\begin{abstract}
Early detection of the self-limiting or chronic tendency towards disease at the beginning of the hepatitis process is a canonical element of the analysis and synthesis to formulate the diagnosis and to decide the most useful and economic therapeutic strategy. Early treatment with antiviral drugs before liver functions undergo deterioration may reduce death and disability especially in young patients.

A formula suggested by a Max Planck equation provides mathematical curves that can be obtained very early during the course of hepatitis using just a few initial serum measurements of the alanine-aminotransferase (ALT) enzyme. The clinical ALT data forming the unpredictable early natural curve of ALT can be transformed into mathematical values capable of providing a deterministic curve that intersects the normal ALT value at what is known as the $G$ point, and allows some predictions to be made concerning the acute or chronic evolution of the disease.

The $G$ point seems to be a good predictor of whether or not a patient with clinically evident first episode of viral hepatitis will develop chronic liver disease as it revealed the initial phase of the first cytolytic episode in 56 untreated patients of our 59 cases; the three wrong predictions involved patients being treated with interferon.
\end{abstract}

Keywords: Liver, hepatitis, alanine-aminotransferase enzyme

\section{INTRODUCTION}

This study is based on the observation that the morphology of the alanine-aminotransferase (ALT) enzyme curves of a first episode of liver viral cytolysis resemble the Max Planck curves describing the relationship between the emission of electromagnetic radiation per unit surface area of a blackbody versus time and wavelength (Herzberg et al, 1945, Planck, 1956, Leconte et al, 1958, Eisberg et al 1974, Tipler, 1977). The Planckian formula suggested a new equation that allows the inclusion of just a few initial

*Corresponding Author: Tel: +39-02-82244501; Fax: +39-02-82244590

** Supported by a grant from Gilette S.p.A., Italy 
serum ALT measurements to produce mathematical curves that closely resemble the natural behaviour of the hepatitis process.

The aim of the study was to investigate whether these curves provide an early prediction of the duration of the first episode of liver viral cytolysis in relation to the temporal threshold of 180 days that defines the chronic evolution of the disease. Such a prediction is useful above all in the case of $\mathrm{C}$ virus related hepatitis which easily evolves towards chronicity. In this case the patients with self-limiting disease would be excluded from therapeutic regimens involving antiviral drugs, whereas those who have a potentially chronic form of the disease need to undergo therapy as early as possible during the initial phase of the process in order to prevent chronic evolution. Since the later treatments are applied the less active they prove to be, information that can put in evidence sufficiently early the needs of the patients and may also achieve a more favourable cost-effectiveness ratio of the antiviral treatment could prove to be very interesting.

\section{PATIENTS AND METHODS}

\section{Human Subjects}

This study was conducted according to the guidelines of the Ethics Committees of the hospitals treating the patients, despite the fact that it could not cause any distress or risk; all of the patients (who were aged 18-58 years) were fully informed.

To avoid exceeding the economic limits imposed by the Diagnosis Related Groups system, and to give a small contribution to private medicine (which is heavily penalised by the inequality of the economic and scientific provisions of the Italian Health Authorities), the serum ALT recordings were strictly limited to those judged to be clinically opportune by the attending physicians without any predetermined times of detection.

The 59 enrolled patients included eight with HAV-correlated hepatitis, 17 with HBV-correlated hepatitis, 28 with $\mathrm{HCV}$-correlated hepatitis (three of whom were being treated with interferon), four with Epstein-Barr virus-correlated hepatitis, one with both HAV and Epstein-Barr virus-correlated hepatitis, and one with cytomegalovirus (CMV)correlated hepatitis. The cases were provided by San Paolo Hospital of Milan (M. Podda), Milan University's Infectious Diseases Clinic (M. Moroni), and the Internal Medicine Department of Melegnano Hospital (G. Benetti).

The study started in February 1993 and ended in February 1998.

\section{Enzyme Assay}

ALT values were measured under normal conditions using a standard method whose normal serum values are less than $40 \mathrm{IU} / \mathrm{ml}$.

\section{Mathematical Method}

The mathematical relationship is expressed by the equation,

$$
y(x)=\frac{a c^{5}}{(x+b)^{5}} \frac{1}{e^{\frac{c}{x+b}}-1}+k
$$

where $e$ is the Eulers number and $k$ a generic constant.

The mathematical curves that can be obtained using this equation are characterized by the following three parameters:

- parameter $a$, which is proportional to the maximum height of the curve;

- parameter $b$, which is proportional to the position of the apex of the curve on the $x$ axis;

- parameter $c$, which is proportional to the width of the curve (Figure 1).

When $x$ is replaced by time $(t)$ expressed in days, $y$ by the value of ALT expressed in IU/ml of serum, and $k$ by the mean normal serum ALT value of $<40 \mathrm{IU} / \mathrm{ml}$ (arbitrarily defined as $30 \mathrm{IU} / \mathrm{ml}$ of serum), equation (2) is obtained,

$$
\operatorname{ALT}(t)=\frac{a \cdot c^{5}}{(t+b)^{5}} \frac{1}{e^{\frac{c}{t+b}}-1}+30
$$




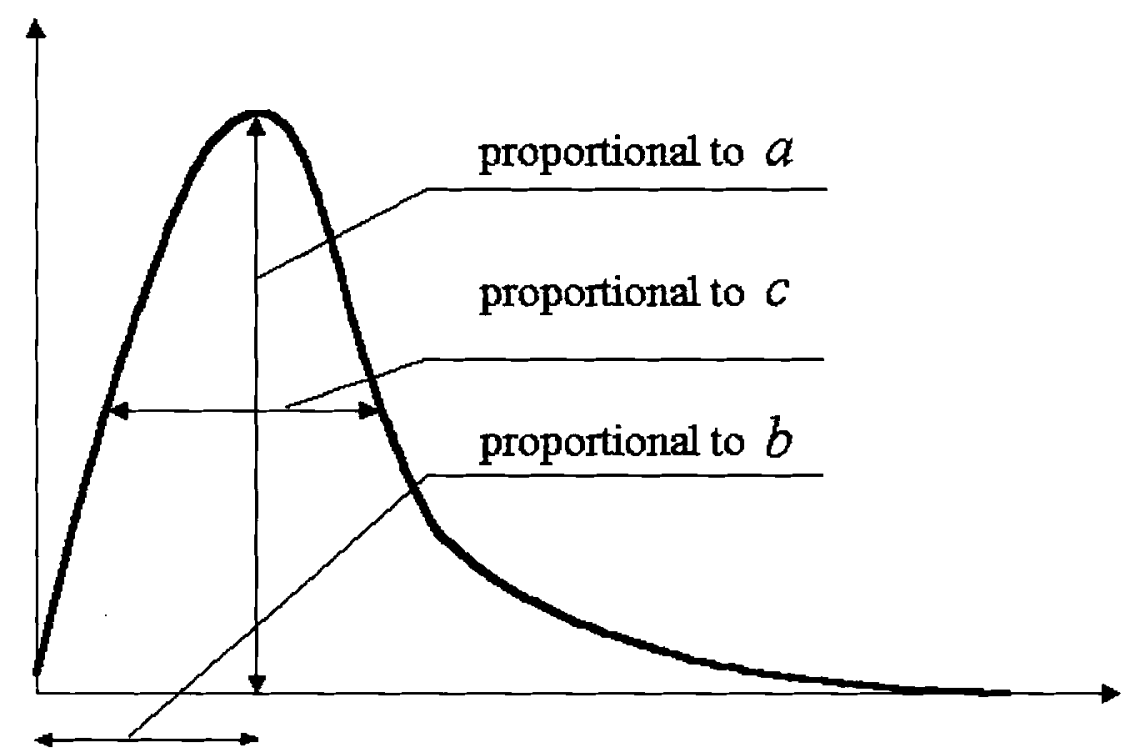

FIGURE 1 Relationship between the parameters $a, b$ and $c$ and the characteristics of the curve.

In this equation, the three parameters $a, b$, and $c$ have a precise medical significance.

Parameter $a$ is dependent on the highest ALT value $\left(\mathrm{ALT}_{\mathrm{M}}\right)$ and is considered to be the index of maximum intensity of the cytolytic process. The mathematical relationship linking parameter $a$ with $\mathrm{ALT}_{\mathrm{M}}$ is expressed by the equation,

$$
\mathrm{ALT}_{\mathrm{M}}=21.2 a+30
$$

where 21.2 is the gradient of the straight line $\left(a, \mathrm{ALT}_{\mathrm{M}}\right)$ graph obtained by numerical calculation, and 30 the arbitrarily defined mean normal serum ALT value of $<40 \mathrm{IU} / \mathrm{ml}$. Parameter $a$ is independent of parameters $b$ and $c$.

Parameter $b$ is dependent on parameter $c$ and is a function of the position in time (days of observation) of the highest ALT value $\left(X_{\text {ALTM }}\right)$.

The mathematical relationship of parameters $b, c$, and $\mathrm{X}_{\mathrm{ALTM}}$ is expressed by the equation,

$$
\mathrm{X}_{\mathrm{ALTM}}=\frac{1}{5} c-b
$$

in which the coefficient of $1 / 5$ has been obtained by numerical calculation and represents the gradient of the straight line $(c, b)$ graph.

Parameter $c$ is the index of the temporal evolution of the simulated process and is dependent on the width of the mathematical curve simulating the process itself. Figure 2 shows that the greater the value of $c$, the wider the curve expressing the length of the episode in the patient under observation.

On the basis of just a few serum ALT measurements, the simulation software written by one of the authors of this paper (A.B.), calculates the parameters $a, b, c$, includes their values in equation (2), and provides the mathematical curve simulating the hepatitis process within a few seconds.

The ideal condition for constructing the mathematical simulations is when data is available from the phase of ascending ALT levels, and there is at least one data point belonging to the phase of descending levels (but better 2-4). In this case, the mathematical curve intersects the line corresponding to the maximum normal serum ALT value of $40 \mathrm{IU} / \mathrm{ml}$ at two points $\left(t_{1}\right.$ and $\left.t_{2}\right)$, which respectively define the beginning and the end of the simulation process (Figure 3). Point $t_{2}$ has been called the $G$ point and, when it lies beyond the conventional threshold of 180 days after the start of the simulation, provides a good prediction of the chronic evolution of the disease. Under these conditions, the curve provides a mathematical simulation of the entire process.

However, a curve can also be constructed using only 3-4 data points from the phase of descending 

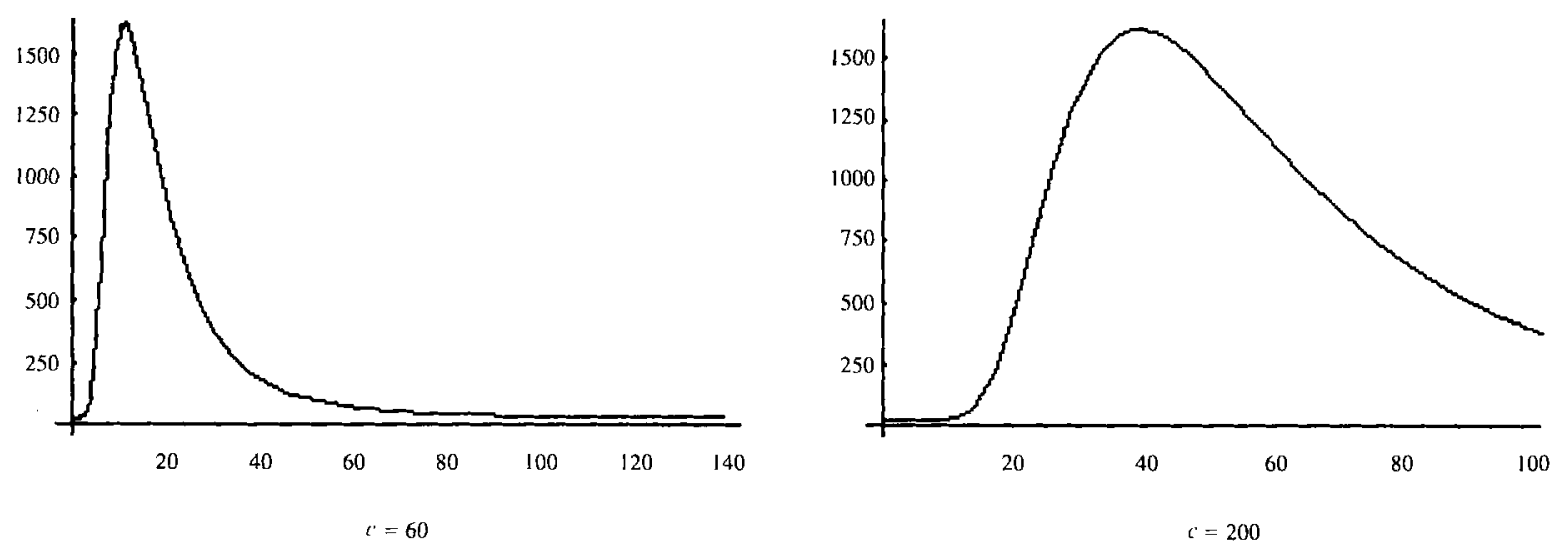

FIGURE 2 Graph of equation (2) showing the parameter $c$ variable. It can be seen that an increase in $c$ leads to an increase in the width of the curve, thus simulating a greater duration of the phenomenon.

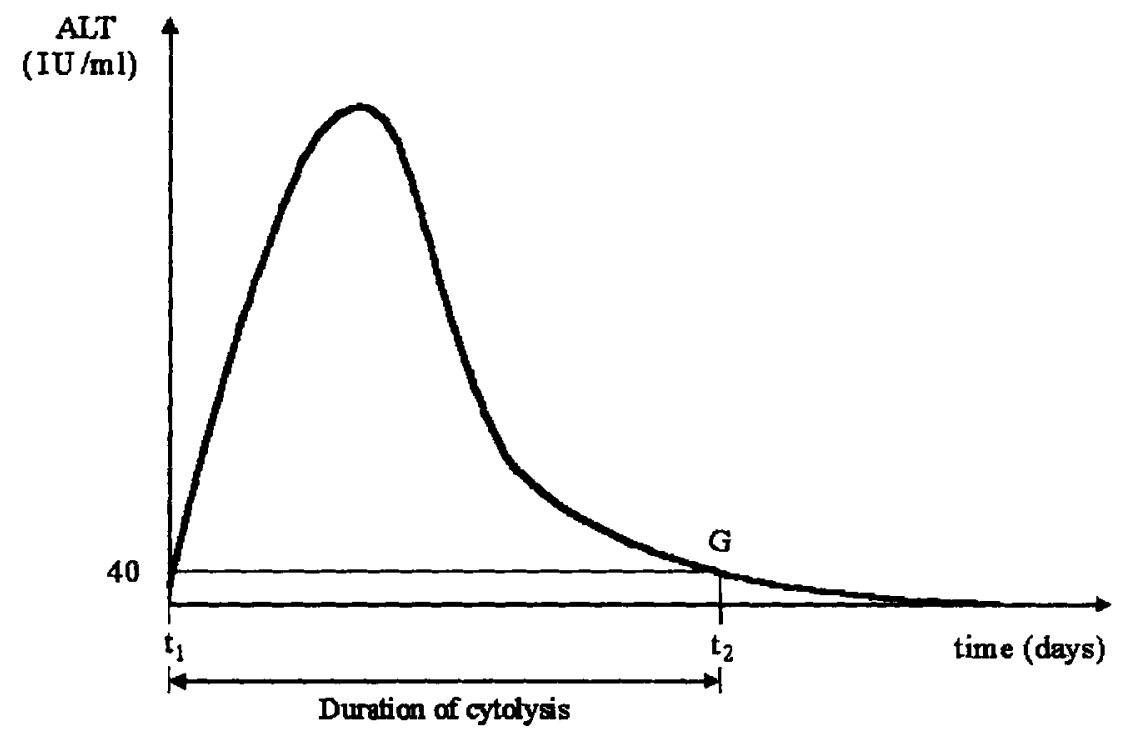

FIGURE 3 The two intersections of the curve on the line indicating a serum ALT value of $40 \mathrm{units} / \mathrm{ml}$ are denoted $t_{1}$ and $t_{2}$. They delimit the duration of the period of above-normal ALT values given by the mathematical simulation. The point $t_{1}$ indicates the theoretical start day of the process and point $t_{2}$ its theoretical end day.

ALT levels. In this case, the mathematical curve simulates the evolution of the process only from the first ALT datum and crosses the normal ALT value line only at the $G$ point. The threshold of 180 days is calculated from the first day of clinical observation.

The following prototypical examples explain the use of the simulation in the two cases.

a) When data before and after the apex of the clinical ALT curve are available. The patient was a 60-year old woman with CMV-dependent hepatitis for whom 11 ALT measurements were available from the beginning to the end of the disease (Table I). The values of the three mathematical parameters of the curve obtained by entering all of the ALT measurements in the simulation software were $a=72, b=7.8$ and $c=94$.

When parameters $a, b$ and $c$ were calculated using the four data points before the peak of the cytolytic process and only one datum after this event, their range of error was approximately $10-11 \%$. The same parameters calculated using an increasing 
TABLE I Patient with CMV-dependent hepatitis

\begin{tabular}{ccc}
\hline Sample & Time (days) & ALT(UL/ml) \\
\hline 1 & 0 & 300 \\
2 & 4 & 760 \\
3 & 9 & 1500 \\
4 & 12 & 1550 \\
5 & 18 & 1100 \\
6 & 23 & 950 \\
7 & 38 & 400 \\
8 & 53 & 185 \\
9 & 73 & 140 \\
10 & 103 & 85 \\
11 & 123 & 45 \\
\hline
\end{tabular}

number of data points until the last led to the error being reduced to nearly zero (Table II).

The use of these values in equation (2) gave the following result,

$$
\operatorname{ALT}(t)=\frac{72 \cdot 94^{5}}{(t+7.8)^{5}} \frac{1}{e^{\frac{94}{t+78}}-1}+30
$$

and led to a mathematical simulation curve overlapping the curve obtained from the clinical serum ALT values (Figure 4). The simulation provided a $G$ point of 135 days; the clinical duration of the disease was about 130 days.

b) When only data from the decreasing phase are available. The patient was a 38-year-old man with HCV-correlated hepatitis whose clinical serum ALT values are shown in Table III. The $G$ point calculated using only the first three ALT
TABLE II The calculated values of the parameters $a, b$, and $c$ obtained from the first five to eleventh value. The maximum error was detected using five ALT values (four before and one after the apex of cytolytic process). The error of the mathematical data versus the clinical data has been calculated on the basis of the differences between the areas subtending the graphs of ALT ( $t$ )

\begin{tabular}{ccccc}
\hline Sample & $a$ & $b$ & $c$ & Error (\%) \\
\hline 5 & 72 & 6,6 & 83 & 10,4 \\
6 & 72 & 7,8 & 94 & 0 \\
7 & 72 & 7,6 & 93 & 0,96 \\
8 & 72 & 7,8 & 93 & 0,96 \\
9 & 72 & 7,8 & 94 & 0 \\
10 & 72 & 7,8 & 94 & 0 \\
11 & 72 & 7,8 & 94 & 0 \\
\hline
\end{tabular}

measurements was 74 days; the $G$ point calculated using an increasing number of data points until the last available measurement was 63 days. The clinical curve shows that the first normal clinical ALT value occurred on day 53.

\section{RESULTS}

The 59 patients were divided into two main groups on the basis of the phase of the disease at the time they were placed under medical care: the first group consisted of 40 patients (three of whom were being treated with interferon) who were observed throughout the course of the disease, and the second of 19 patients who were only seen during the decreasing
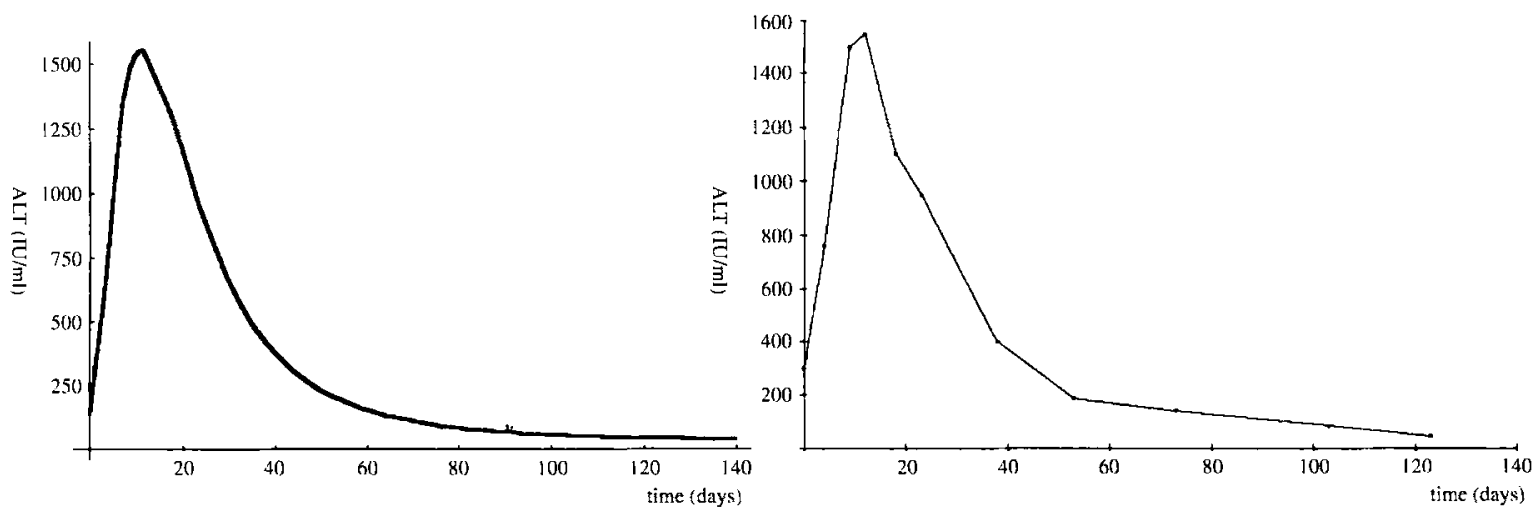

FIGURE 4 (Left) Graphic representation of the mathematical curve provided by equation 2 . The parameters $a, b$, and $c$ were calculated from the 11 data points reported in Table $\mathbf{U}$. (Right) The clinical curve provided by the wild ALT serum records. 
TABLE III Patient with HCV-correlated hepatitis

\begin{tabular}{ccc}
\hline \multicolumn{3}{c}{ Initial ALT Determinations } \\
\hline Sample & Time (days) & ALT (UI/ml) \\
\hline 1 & 0 & 2240 \\
2 & 4 & 1760 \\
3 & 9 & 558 \\
4 & 12 & 550 \\
\hline \multicolumn{3}{c}{} \\
\hline Sample & Following ALT Determinations \\
\hline 5 & Time (days) & ALT (UI/mi) \\
6 & 18 & 281 \\
7 & 23 & 76 \\
8 & 38 & 50 \\
\hline
\end{tabular}

phase of the cytolytic process. The results are presented in terms of the acute or chronic clinical evolution of the first disease episode on the basis of the incidence of $G$ point values over the conventional 180-day threshold.

Table IV summarizes the results obtained in the 37 patients for whom ALT data relating to both the ascending and the initial descending phases of the ALT curve were available. In 22 of these cases, the duration of clinically high ALT levels was less than 180 days: the mean $G$ point was $102.95 \pm 38.2$ days (range: $34-177$ days), and the mean time of prediction was $25.5 \pm 12.3$ days (range: 7-60 days). In the remaining 15 patients, the duration of high ALT levels was more than 180 days, with a mean $G$ point of $399.26 \pm 161.18$ days (range: 229-747 days), and a mean time of prediction of $49.3 \pm 19.9$ days (range: $30-96$ days).

Table $\mathrm{V}$ summarizes the results obtained in the 19 patients admitted to hospital during the descending phase of the ALT curve. Fifteen of these cases had clinically high ALT levels for a period of less than 180 days, with a mean $G$ point of $95.26 \pm 40.87$ days (range: $49-172$ days) and a mean time of prediction of $25.86 \pm 9.21$ days (range: 12-42 days); in the remaining four patients, the period of high ALT levels was more than 180 days, with a mean $G$ point of $403.50 \pm 123.65$ days (range: $219-483$ days) and a mean time of prediction of $43 \pm 9.59$ days (range: 34-54 days).

Table VI shows the baseline characteristics of the patients for whom data relating to the ascending and descending phases of the ALT curves were available, and Table VII the baseline characteristics of the patients for whom only ALT data of the descending phase were available.

Table VIII shows the data relating to the three patients in whom the prediction failed because they were being treated with interferon therapy.

TABLE IV Results obtained in the 37 patients for whom ALT data relating to both the ascending and the initial descending phases of the ALT curve were available

\begin{tabular}{|c|c|c|c|c|c|c|}
\hline \multirow{2}{*}{\multicolumn{2}{|c|}{ Patients }} & \multicolumn{3}{|c|}{ ALT Determinations } & \multirow[t]{2}{*}{ G point* } & \multirow[t]{2}{*}{ Clinical Evolution } \\
\hline & & Before Peak & After Peak & $\overline{\text { Days Elapsed }}$ & & \\
\hline \multirow{2}{*}{37} & 22 & $1,9 \pm 1$ & $3,3 \pm 1$ & $25,5 \pm 12,3$ & $102,95 \pm 38,2$ & acute \\
\hline & 15 & $2,7 \pm 1,7$ & $3,1 \pm 1,1$ & $49,3 \pm 19,9$ & $399,26 \pm 161,2$ & chronic \\
\hline
\end{tabular}

*expressed in days

TABLE V Results obtained in the patients admitted to hospital during the descending phase of the ALT curve

\begin{tabular}{cccccc}
\hline Patients & $\begin{array}{c}\text { Number of ALT } \\
\text { Determinations }\end{array}$ & Days Elapsed & G point* & $\begin{array}{c}\text { Clinical } \\
\text { Evolution }\end{array}$ \\
\hline \multirow{2}{*}{19} & 15 & $4,5 \pm 1,24$ & $25,9 \pm 9,2$ & $95,3 \pm 40,9$ & acute \\
& 4 & $4,3 \pm 0,5$ & $43 \pm 9,6$ & $403,5 \pm 123,6$ & chronic \\
\hline
\end{tabular}

*expressed in days 
TABLE VI Baseline characteristics of the patients for whom data relating to the ascending and descending phases of the ALT curves were available

\begin{tabular}{|c|c|c|c|c|c|c|c|}
\hline \multirow[t]{2}{*}{ Patient } & \multirow[t]{2}{*}{ Age/Sex } & \multirow[t]{2}{*}{ Virus } & \multicolumn{3}{|c|}{ Number of ALT Determination } & \multirow[t]{2}{*}{ G point* } & \multirow[t]{2}{*}{ Clinical Evolution } \\
\hline & & & Before Peak & After Peak & Days Elapsed & & \\
\hline 1 & $18 / \mathrm{m}$ & HAV & 1 & 3 & 7 & 34 & acute \\
\hline 2 & $43 / f$ & HAV & 2 & 3 & 29 & 177 & acute \\
\hline 3 & $18 / \mathrm{m}$ & HAV & 1 & 4 & 29 & 83 & acute \\
\hline 4 & $21 / \mathrm{m}$ & HAV & 2 & 3 & 21 & 86 & acute \\
\hline 5 & $25 / \mathrm{m}$ & HAV & 1 & 4 & 10 & 78 & acute \\
\hline 6 & $28 / \mathrm{m}$ & HBV & 1 & 3 & 31 & 117 & acute \\
\hline 7 & $51 / \mathrm{m}$ & HBV & 1 & 3 & 38 & 163 & acute \\
\hline 8 & $30 / \mathrm{m}$ & HBV & 3 & 3 & 7 & 94 & acute \\
\hline 9 & $40 / f$ & $\mathrm{HBV}$ & 1 & 4 & 21 & 115 & acute \\
\hline 10 & $45 / \mathrm{m}$ & HBV & 1 & 4 & 41 & 89 & acute \\
\hline 11 & $29 / \mathrm{f}$ & $\mathrm{HBV}$ & 1 & 3 & 15 & 90 & acute \\
\hline 12 & $47 / \mathrm{m}$ & $\mathrm{HBV}$ & 4 & 2 & 19 & 146 & acute \\
\hline 13 & $30 / f$ & HBV & 1 & 4 & 30 & 147 & acute \\
\hline 14 & $22 / f$ & HBV & 2 & 3 & 16 & 77 & acute \\
\hline 15 & $35 / f$ & $\mathrm{HCV}$ & 1 & 3 & 22 & 117 & acute \\
\hline 16 & $37 / \mathrm{f}$ & $\mathrm{HCV}$ & 3 & 2 & 35 & 129 & acute \\
\hline 17 & $41 / f$ & $\mathrm{HCV}$ & 3 & 3 & 35 & 124 & acute \\
\hline 18 & $29 / \mathrm{f}$ & $\mathrm{HCV}$ & 2 & 4 & 26 & 70 & acute \\
\hline 19 & $49 / \mathrm{m}$ & $\mathrm{HCV}$ & 3 & 2 & 30 & 89 & acute \\
\hline 20 & $25 / \mathrm{m}$ & $\mathrm{HCV}$ & 2 & 3 & 60 & 25 & acute \\
\hline 21 & $25 / \mathrm{f}$ & EBV & 2 & 3 & 15 & 80 & acute \\
\hline 22 & $60 / f$ & $\mathrm{CMV}$ & 4 & 7 & 25 & 135 & acute \\
\hline 1 & $35 / \mathrm{f}$ & HBV & 3 & 2 & 40 & 362 & chronic \\
\hline 2 & $21 / \mathrm{m}$ & $\mathrm{HBV}$ & 3 & 2 & 31 & 447 & chronic \\
\hline 3 & $25 / \mathrm{m}$ & $\mathrm{HCV}$ & 1 & 4 & 30 & 267 & chronic \\
\hline 4 & $27 / \mathrm{m}$ & $\mathrm{HCV}$ & 4 & 2 & 41 & 556 & chronic \\
\hline 5 & $56 / \mathrm{m}$ & $\mathrm{HCV}$ & 8 & 2 & 96 & 345 & chronic \\
\hline 6 & $29 / \mathrm{m}$ & $\mathrm{HCV}$ & 1 & 5 & 47 & 229 & chronic \\
\hline 7 & $39 / \mathrm{m}$ & $\mathrm{HCV}$ & 2 & 4 & 45 & 303 & chronic \\
\hline 8 & $31 / \mathrm{m}$ & $\mathrm{HCV}$ & 4 & 1 & 46 & 731 & chronic \\
\hline 9 & $50 / \mathrm{f}$ & $\mathrm{HCV}$ & 2 & 3 & 39 & 243 & chronic \\
\hline 10 & $58 / \mathrm{f}$ & $\mathrm{HCV}$ & 3 & 4 & 96 & 407 & chronic \\
\hline 11 & $23 / \mathrm{m}$ & $\mathrm{HCV}$ & 2 & 3 & 51 & 747 & chronic \\
\hline 12 & $26 / \mathrm{m}$ & $\mathrm{HCV}$ & 3 & 4 & 42 & 270 & chronic \\
\hline 13 & $24 / m$ & $\mathrm{HCV}$ & 2 & 3 & 47 & 350 & chronic \\
\hline 14 & $26 / \mathrm{m}$ & $\mathrm{HCV}$ & 1 & 4 & 50 & 368 & chronic \\
\hline 15 & $40 / \mathrm{m}$ & $\mathrm{HCV}$ & 2 & 3 & 39 & 364 & chronic \\
\hline
\end{tabular}

*expressed in days

\section{DISCUSSION}

The various types of viral hepatitis are considered endemic throughout the world and even hyperendemic in some areas. Cirrhosis, the chronic sequela of many forms of hepatitis, involves considerable social and financial costs, poses a number of ethical problems, leads to the loss of a large number of working hours and remains one of the most frequent causes of death. Given that the cost of treating both the acute and chronic phase of the unvaccinable forms of hepatitis is destined to rise, there is growing interest in the possibility of being able to distinguish as early as possible the patients with self-limiting hepatitis that do not need it. On the contrary in an attempt to stop the chronical evolution of the disease the patients affected by a potentially chronic form need to undergo antiviral treatments 
TABLE VII Baseline characteristics of the patients for whom only ALT data of the descending phase were available

\begin{tabular}{|c|c|c|c|c|c|c|}
\hline \multirow[t]{2}{*}{ Patient } & \multirow[t]{2}{*}{ Age/Sex } & \multirow[t]{2}{*}{ Virus } & \multicolumn{2}{|c|}{ Number of ALT Determination } & \multirow[t]{2}{*}{ G point* } & \multirow[t]{2}{*}{ Clinical Evolution } \\
\hline & & & After Peak & Days Elapsed & & \\
\hline 1 & $30 / \mathrm{m}$ & HAV & 5 & 14 & 79 & acute \\
\hline 2 & $26 / f$ & HAV & 3 & 16 & 64 & acute \\
\hline 3 & $32 / \mathrm{f}$ & HAV & 4 & 29 & 83 & acute \\
\hline 4 & $32 / \mathrm{f}$ & $\mathrm{HBV}$ & 4 & 29 & 146 & acute \\
\hline 5 & $25 / \mathrm{f}$ & HBV & 5 & 41 & 115 & acute \\
\hline 6 & $36 / \mathrm{f}$ & HBV & 3 & 20 & 134 & acute \\
\hline 7 & $41 / f$ & HBV & 4 & 29 & 172 & acute \\
\hline 8 & $39 / \mathrm{f}$ & $\mathrm{HBV}$ & 4 & 25 & 155 & acute \\
\hline 9 & $29 / \mathrm{m}$ & $\mathrm{HCV}$ & 4 & 42 & 80 & acute \\
\hline 10 & $38 / \mathrm{m}$ & $\mathrm{HCV}$ & 8 & 12 & 63 & acute \\
\hline 11 & $35 / f$ & $\mathrm{HCV}$ & 5 & 30 & 114 & acute \\
\hline 12 & $18 / \mathrm{m}$ & EBV & 5 & 36 & 50 & acute \\
\hline 13 & $39 / \mathrm{m}$ & EBV & 4 & 18 & 55 & acute \\
\hline 14 & $41 / \mathrm{m}$ & EBV & 4 & 26 & 49 & acute \\
\hline 15 & $28 / \mathrm{m}$ & $\mathrm{HAV}+\mathrm{EBV}$ & 6 & 21 & 70 & acute \\
\hline & & & & & & acute \\
\hline 1 & $30 / f$ & HBV & 4 & 48 & 219 & chronic \\
\hline 2 & $25 / \mathrm{m}$ & $\mathrm{HCV}$ & 5 & 54 & 455 & chronic \\
\hline 3 & $23 / \mathrm{m}$ & $\mathrm{HCV}$ & 4 & 34 & 457 & chronic \\
\hline 4 & $37 / \mathrm{m}$ & $\mathrm{HCV}$ & 4 & 36 & 483 & chronic \\
\hline
\end{tabular}

*expressed in days

TABLE VIII Data relating to the three patients in whom the prediction failed because they were being treated with interferon therapy

\begin{tabular}{lcccccc}
\hline Patient & Age/Sex & Virus & \multicolumn{3}{c}{ ALT Determination } & G point* \\
\cline { 5 - 7 } & & & Before Peak & After Peak & Days Elapsed* & \\
\hline 1 & $35 / \mathrm{m}$ & HCV & 2 & 4 & 60 & 25 \\
2 & $41 / \mathrm{m}$ & HCV & 1 & 3 & 24 & 89 \\
3 & $39 / \mathrm{f}$ & HCV & 2 & 3 & 30 & 55 \\
\hline
\end{tabular}

*expressed in days

during the initial phases of the process without waiting 6-9 months, even if the liver functions are not decompensated or decreased. This is urgent especially in young patients because: a) they are more sensitive to the antiviral treatment; b) despite the natural history, the information about the long-term clinical and economical outcome of chronic hepatitis remains controversial, the suggestion that it is a life-shortening disease is an unquestionable fact; c) long-term interferon treatment, despite its high cost and failures in many patients, prolongs life expectancy intrinsically reduced by the course of the disease; d) the only exclusion criterion of the therapeutic intervention is very low grade of inflammation. The combination of these four conditions remains a very intriguing and disquieting problem not only for physicians but also for the most imperturbable economist (Buti, 1998; Koff, 1998; Messori, 1998).

In brief, the possibility of making such a dichotomy between treatable and untreated patients, considered not feasible up till today, could avoid the indiscriminate use of some treatments during the acute phase of the disease as well as the uneconomic wait until the passing of the threshold time indicating chronic disease. 
However, despite our increasing understanding of the various types of viral and toxic hepatitis, there is still no method that is capable of providing circumstantial evidence concerning their acute or chronic destiny. The temporal determination of ALT levels alone is not predictive because the chronic evolution of these diseases can currently only be revealed $a$ posteriori by detecting the nonnormalization of serum ALT levels 6-12 months after an acute episode or the non-clearance of hepatitis viruses due to their persistent replication up to one year after infection (both of which require expensive analytical methods), or by detecting hepatocyte necrosis, inflammation and fibrosis at liver biopsy.

This study started from the fact that the evolution of many natural phenomena (Fermi, 1966, Weiss, 1994, Jensen et al, 1995, Sherman, 1995, Wise et al, 1995), including the behaviour of ALT levels during hepatitis, recalls the morphology of Planckian curves. This observation led to the writing of a formula (derived from the Planck equation) that produces curves using just a few data points measured during the days after the highest ALT value is reached. The curves cross the line of normal ALT values at what is called the $G$ point on the temporal axis, which seems to predict well in advance whether the initial phase of the disease will last longer than the 180 days considered to be the threshold of chronicity.

The prediction curves can be constructed using a minimum number of three data points measured at the beginning of the descending phase of the disease. The $G$ point obtained from these simulations cannot predict the definitive acute conclusion of HCVdependent hepatitis when it indicates a simulated duration of less than 180 days because the disease may reemerge after a first short and only apparently conclusive episode; however, it does seem to predict chronic evolution if it indicates a duration of more than 180 days.

The $G$ point provided a good prediction of the acute or chronic destiny of the first cytolytic episode in 56 of our 59 cases; the three wrong predictions all involved patients being treated with interferon.
This paper cannot go beyond making a preliminary proposal that takes the parameters $a$ and $c$ provided by equation (2) as numerical markers predicting the behaviour of necrosis: parameter $a$ provides an index of its maximal intensity and parameter $c$ an index of its possible temporal evolution.

Mathematical representations of ALT behaviour during hepatitis can be obtained using other statistical functions, such as $\gamma-$ and log-normal distribution, but the fact that these functions only provide dependent parameters makes it more difficult to obtain indices that separately represent the severity and duration of the cytolytic event; furthermore, they assign meanings and virtues that are different from those provided by the formula proposed in this study.

The independence of parameters $a, b$ and $c$ that allows the empirical temporal ALT curve to be transformed into a semi-deterministic mathematical curve provides insights into the stochastic behaviour of hepatitis processes that go beyond the critical horizon offered by direct observation during the early disease phase. It can thus be hypothesised that a few early ALT measurements are capable of providing information concerning the initial conditions determining the evolution of the process, and that this information determines the predictive power of the formula to give an early indication as to whether the disease may evolve chronically. If this virtue is confirmed, it may become possible to propose its use for the study of other kinds of non-linear stochastic medical and non-medical phenomena that are characterized by behaviours whose trajectories are similar to the Planckian curve.

\section{References}

Buti, M., Casado, M. A., Fosbrook, L. and Esteban, R. (1998) Cost effectiveness of the treatment of chronic hepatitis $C$ with interferon-alpha. Gastroenterol Hepatol; 21(4), 161-168.

Eisberg, R. and Resnick, R. (1974) Quantum Physics of Atoms, Molecules, Solids, Nuclei and Particles. J. Whiley \& Sons.

Fermi, E. (1966) Molecular Crystal and Quantum Statistics. Lloyd Motz, W.A. Benjamin Inc;.

Herzberg, G. (1945) Infrared and Raman Spectra of Polyatomic Molecules. D. Van Nostrand Company Inc.

Koff, R. S. (1998) Therapy of hepatitis C: cost-effectiveness analysis. Hepatology; 26(3 Suppl 1), 152S-155S. 
Jensen, J. B., Egsgaard, H., Van Onckelen, H. and Jochinsen, B. U. (1995) Catabolism of indole-3-acetic acid and 4 and 5 chloroindole-3-acetic acid in Bradyrzolium japonicum. J Bacteriol; 141(2), 5762-6.

Leconte, J. (1958) Spectroscopie dans l'infrarouge. Encyclopedia of Physics, XXVI, Light and matter. Springer \& Verlag.

Messori, A. (1998) Cost-effectiveness of interferon in chronic myeloid leukaemia: analysis of four clinical studies. Ann Oncol; 9(4), 389-396.

Planck, M. (1956) Autobiografia scientifica ed ultimi saggi. Ed. Scientifiche Einaudi.
Sherman, J. D. and Stack, S. M. (1995) Two-dimensional spread of synoptonemal complexes from solanaceus plants. VI. Highresolution recombination nodule map for tomato (Licopersicon esculantum). Genetics; 141(2), 683-708.

Tipler, P. A. (1977) Modern Physics. Worth Pubblischer Inc.

Weiss, G. H. (1994) A Primer of Random Walkology in Fractal and Science. Springer Verlag;

Wise, T. and Klindt, J. (1995) Thymic weight changes endocrine relationships during maturation in cattle: effects of age, sex and castration. Growth Dev Aging; 59(3), 139-48. 


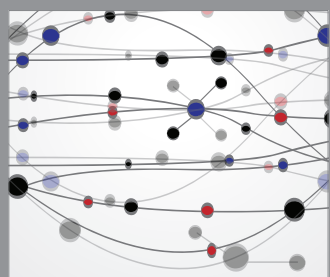

The Scientific World Journal
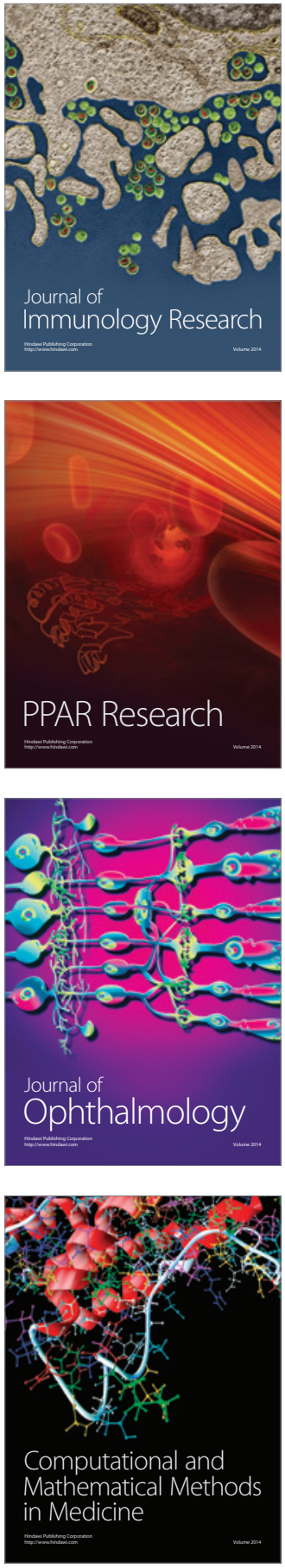

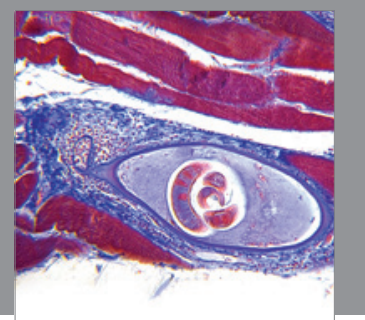

Gastroenterology

Research and Practice
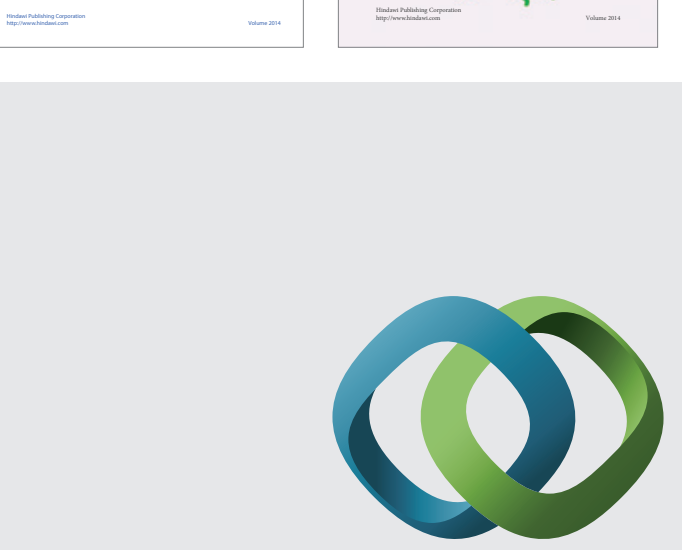

\section{Hindawi}

Submit your manuscripts at

http://www.hindawi.com
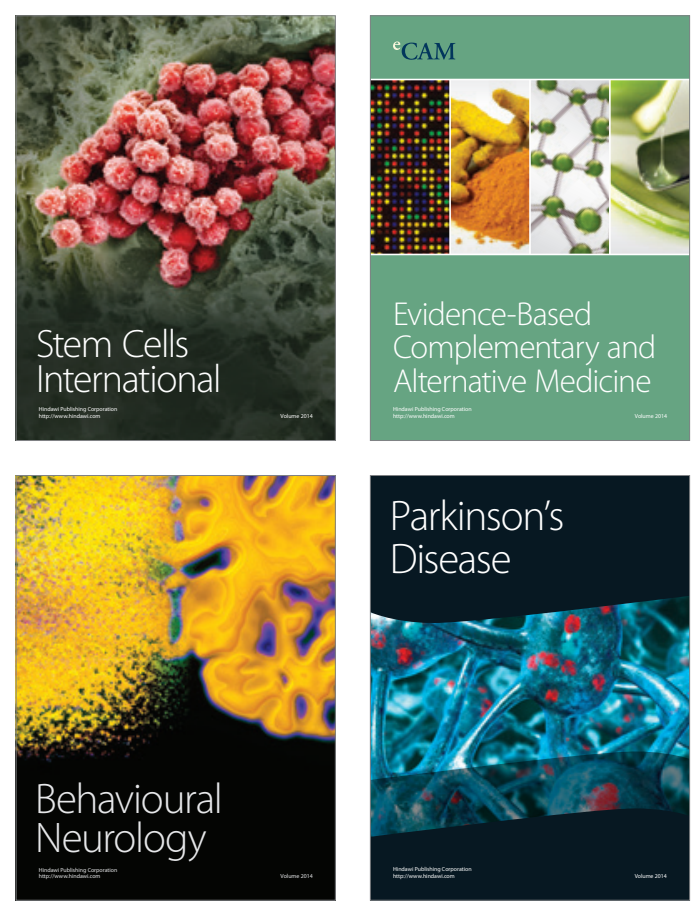

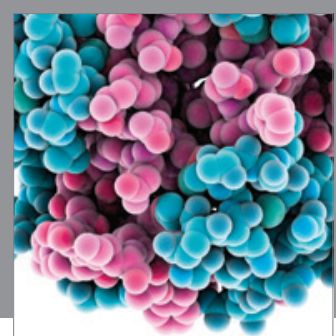

Journal of
Diabetes Research

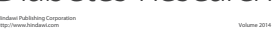

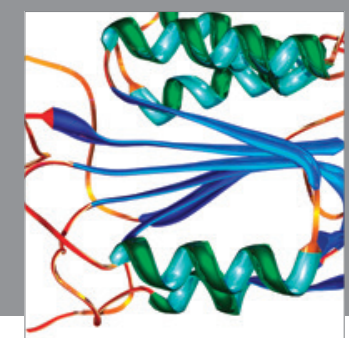

Disease Markers
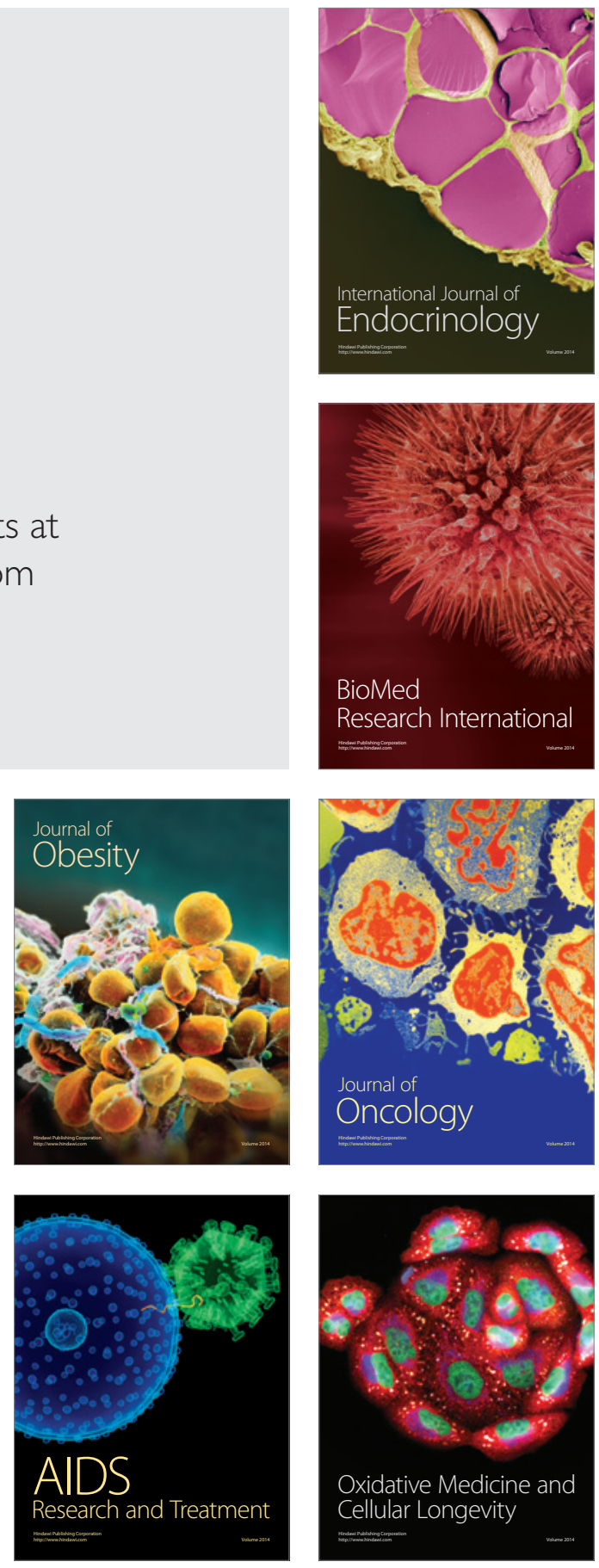